\title{
Computationally Analyzing Biometric Data and Virtual Response Testing in Evaluating Learning Performance of Educational Setting Through
}

\section{SIGRADI2018 TECHNOPOLITICAS \\ xxii congresso da sociedade iberoamericana de gráfica digital 22th conference of the iberoamerican society of digital graphics 07|08|09|novembro|2018 iau usp | são carlos | sp br}

\author{
Saleh Kalantari \\ Cornell University | U.S.A. | sk3268@cornell.edu \\ Jesus Cruze-Garza \\ University of Houston | U.S.A. | jgcruz@central.uh.edu \\ Pamela Banner \\ Yale University | U.S.A. | pamela.banner@yale.edu \\ Jose Luis Contreras-Vidal \\ University of Houston | U.S.A. | jlcontr2@central.uh.edu
}

\begin{abstract}
Due to construction costs, the human effects of innovations in architectural design can be expensive to test. Post-occupancy studies provide valuable data about what did and did not work in the past, but they cannot provide direct feedback for new ideas that have not yet been attempted. This presents designers with something of a dilemma. How can we harness the best potential of new technology and design innovation, while avoiding costly and potentially harmful mistakes? The current research use virtual immersion and biometric data to provide a new form of extremely rigorous human-response testing prior to construction. The researchers' hypothesis was that virtual test runs can help designers to identify potential problems and successes in their work prior to its being physically constructed. The pilot study aims to develop a digital pre-occupancy toolset to understand the impact of different interior design variables of learning environment (independent variables) on learning performance (dependent variable). This project provides a practical toolset to test the potential human impacts of architectural design innovations. The research responds to a growing call in the field for evidence-based design and for an inexpensive means of evaluating the potential human effects of new designs. Our research will address this challenge by developing a prototype mobile brain-body imaging interface that can be used in conjunction with virtual immersion.
\end{abstract}

Keywords Signal Processing; Brain; EEG; Virtual Reality; Big Data; Learning Performance

\section{INTRODUCTION}

The idea of studying behavioral patterns to investigate human responses to architectural design has been around for many years, but it is only recently that this approach has consolidated into the widely recognized paradigm known as evidence-based design (EBD). This approach to architectural design relies on the careful empirical study of human responses and outcomes to inform design decisions (Cama, 2009; Edelstein \& Macagno, 2012; Hamilton \& Watkins, 2009). Many previous investigations have provided evidence that EBD practices can successfully improve the overall perceived quality of the architectural environment as well as specific measures of building performance (Ulrich, 2001, 2006; Hamilton \& Watkins, 2009; Sailer, 2009; Lawson, 2010). The EBD approach has become particularly influential in healthcare settings, where it has been associated with improvements in the quality of care, greater patient satisfaction, and a decrease in the number of medical errors (Ulrich et al., 2008).

Current technology encourages designers to introduce more innovation into their work. While this innovation often leads to exciting and effective results, it also takes us away from tried-and-true solutions, into relatively uncharted territory. Scholars have demonstrated that the characteristics of the built environment can have a significant effect on human well-being. Specific design components have been strongly correlated with health outcomes (Truong \& Ma, 2006; Wheaton et al., 2015), as well as with human efficiency and productivity (Day, 2017). Renewed interest in human-centered design in recent decades has led researchers to document the contributions of architectural design for reducing stress, improving mood, and enhancing visual memory, among other benefits (Ulrich et al., 1991; Sallis et al., 2006). Numerous studies have investigated different architectural styles and design-choices and how they affect human 
experiences (Choo et al., 2017; Vecchiato et al., 2015; Vartanian et al., 2013; Roe et al., 2013; Banaei et al., 2015; Shin et al., 2014; Küller et al., 2009).

Unfortunately, when innovative designs are created, it is difficult to accurately evaluate their full human effects, positive or negative, until after the buildings are constructed and put into use. This presents contemporary designers with a dilemma. How can we harness the best potential of the innovation allowed by today's technology, while avoiding costly and potentially harmful mistakes?

The goal of this research was to examine the effects of building-design on human factors (stress, anxiety, visual memory, etc.), by measuring the responses of participants as they interact with different architectural designs using Virtual Reality technology. The researchers' hypothesis was that virtual "test runs" can help designers to identify potential problems and successes in their work prior to its being physically constructed.

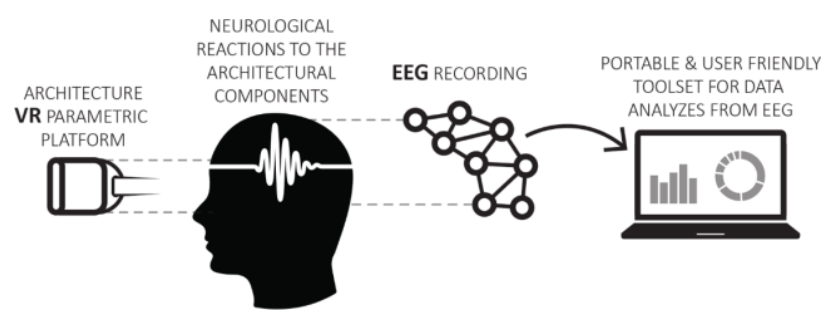

Figure 1: the description should be located below the figure, Arial 8. Lee and Lee (1991) rated the paper as "the best in its field" (p.14)

The learning environment includes social, cultural, temporal, physical (built and natural), and sometimes virtual aspects (McGregor, 2004; OECD, 2014). Student performance has been shown to have a significant relationship to the quality of the learning environment (Chan \& Richardson, 2005). Poor-quality environments can create barriers such as impaired concentration, boredom, and claustrophobia (Mendell \& Heath, 2005), and thereby lead to poorer educational outcomes. A highquality learning environment, in contrast, supports engagement and inquiry, and accounts for a diverse range of developmental needs, learning styles, and abilities (Martin, 2010). Despite the well-established link between learning environments and student outcomes, the specific elements within these environments that affect students have not been rigorously broken down and empirically investigated. This is especially true in relation to the architectural environment. Temple (2007) notes that, "Where connections between the built environment and educational activities are made, the basis for doing so tends to be casual observation and anecdotes rather than firm evidence." Further research is needed to help identify the individual elements of the physical environment that might be important from a design perspective in order to help support student achievement. (Kaup et al., 2013; Barret et al., 2015). The work that has been done in this area suggests, at best, a number of general themes regarding the optimal design of learning spaces. Perhaps the most dominant theme is that these spaces need to be flexible, both pedagogically and physically, so that they can be adjusted to reflect the nuances of different knowledge areas and specializations, as well as different learning styles (Butin, 2000). This awareness reflects the growing understanding among teachers of the importance of active and collaborative learning, student-faculty interaction, enriching educational experiences, and opportunities for intellectual creativity. Along with this emerging new pedagogy comes an increased interest in transforming traditional classrooms to a new learning environment that can more easily accommodate collaborative and active learning in a technology-rich setting (Brooks et al., 2012).

Other specific factors that have been associated with higher student performance in the existing literature include the incorporation of naturalness (in light, sound, temperature, air quality, and links to nature) (Crandell \& Smaldino, 2000; Daisey et al., 2003; Wargocki \& Wyon, 2007; Barret et al., 2015); learning environments that create a greater sense of individuality, ownership, and flexibility (Zeisel et al., 2003; Ulrich, 2004; Barret et al., 2015); and environments that provide greater stimulation and sensory impact (Kuller et al., 2009; Fisher et al., 2014; Barret et al., 2015). As can be seen in the dating of these citations, this is a relatively new area of study, and there is a lot of hope in the literature that future investigations can help to further isolate the relevant factors and contribute to learning outcomes by implementing these concepts and techniques.

\section{EXPERIMENTAL DESIGN AND PROCEDURE}

The researchers' primary objective was to create a standardized and intuitive toolset that can be used by designers to help evaluate their work. Electroencephalography (EEGs) will be used, along with other noninvasive biophysical measurements and selfreporting, to objectively analyze the participants' conscious and subconscious responses to different building designs.

We collected brain activity and relative spatial location from the participants that elect to wear the EEG headset. We also collected voluntarily self-reported non-identifiable information such as age, gender, race, ethnicity, whether the participants consume caffeine, alcohol or recreational drugs, whether they have, or have had in the past stroke, concussion, seizures, movement disorders or other neurological or physical conditions, the participant's current occupation.

Our intention with the various measurements was twofold: to quantify the human stress response and assess performance on a number of cognitive tasks. Based on previous studies examining the first of these (Healey and Picard, 2005), we incorporated three biometrics associated with the highest correlation to self-reported stress-electrocardiography (ECG), galvanic skin response (GSR), and electroencephalography (EEG). Additionally, an accelerometer and electrooculography (EOG) sensors were attached to or near the EEG cap to track head and eye movement in each environment. All data was collected at $500 \mathrm{~Hz}$ and synchronized using the 64-channel ActiCHamp module (Brain Products $\mathrm{GmbH}$, Germany) with $\mathrm{Ag} / \mathrm{AgCl}$ active electrodes. A total of 63 electrodes were used (57 for EEG arranged according to the international 10-20 placement system, 4 for EOG and 

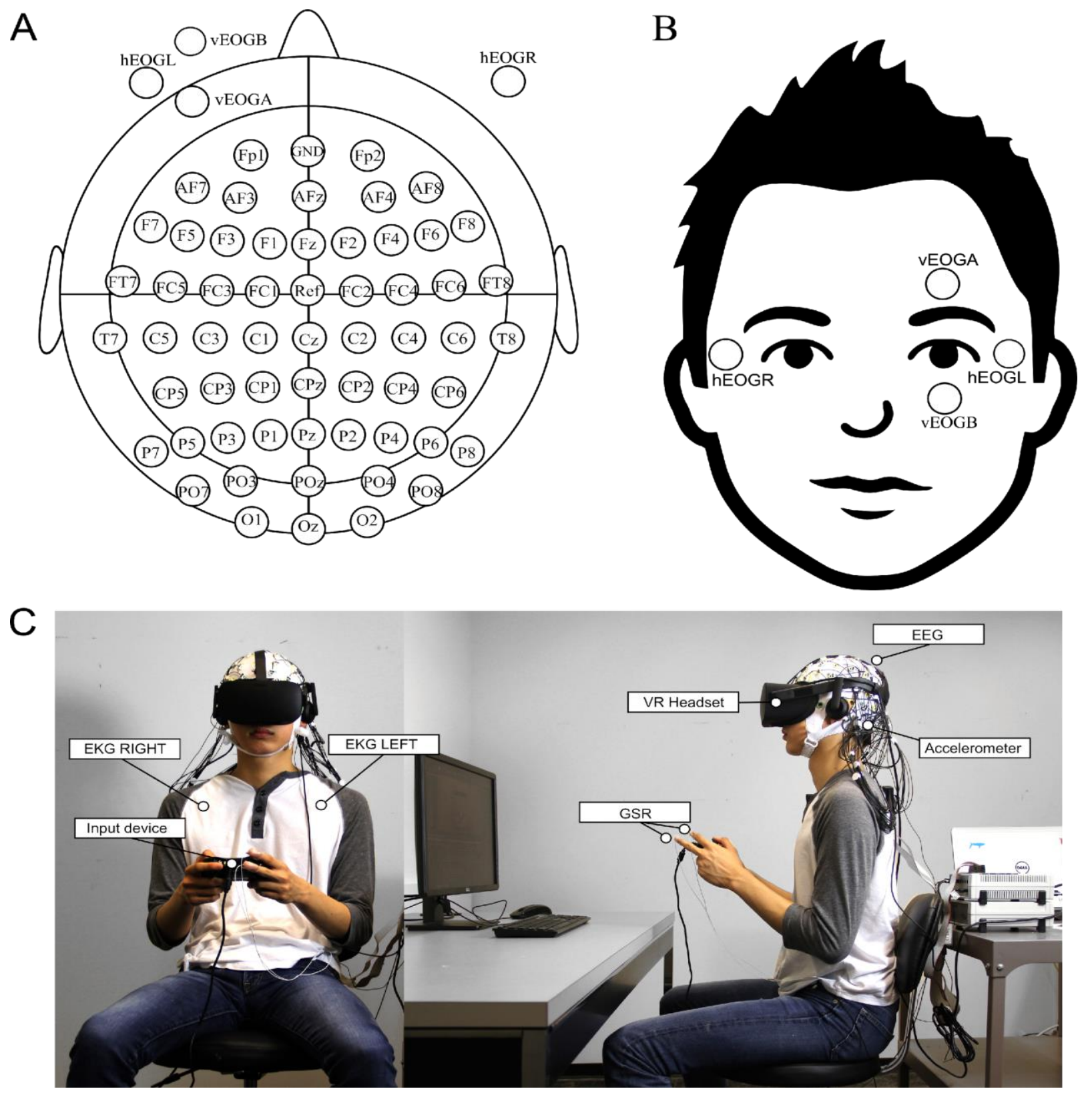

Figure 2: The experimental setup for data recording: (a) EEG electrode locations, (b) EOG electrode locations, and (c) all sensor equipment as worn by a study participant. Source: authors.

2 for ECG). The impedance of each electrode was kept below $50 \mathrm{k} \Omega$, and often below $20 \mathrm{k} \Omega$, at all times. This was ensured throughout the study with careful placement of the virtual reality headset. Figure 2 shows the electrode and equipment placement on a study participant.

The data was recorded using the BrainVision Recorder software (Brain Products GmbH, Germany) and synchronized to the participants' responses and the virtual reality environment using the Lab Streaming Layer program (Kothe 2014). Prior to entering each new room iteration or segment of the experiment, participants were prompted to press a specific button, programmed to act as a marker on the recorded biometric data. Screen recordings were also collected throughout the study.

Following an introductory survey and neutral baseline recordings, the study was segmented into two main parts. Experiment "A" shown in blue in Figure 2 consisted of five memory tasks-the Benton Test, Visual Memory Test,
Stroop Task, Digit Span Task and a mathematical problem-solving task-followed by self-reported stress and mental fatigue on a 10-point Likert scale. Each task was either consistently timed or a pre-determined number of questions to ensure homogeneity between room conditions. Instructions were provided prior to beginning the study for participants unfamiliar with the tasks. Participants were asked to complete the same tasks in a real classroom, a VR representation of it, and in nine other classroom iterations. Experiment "B" shown in red in Figure 2 initially asked subjects to navigate along a preselected path through a cityscape featuring __ buildings with unique facades, after which they were asked questions regarding what they remember of the path they took. This was repeated once more so that participants could navigate the cityscape knowing the type of memorization questions that would be asked of them. Finally, they were instructed to design the façade of the "ideal landmark in their favorite city" by modifying characteristics such as height, base geometry and twist 


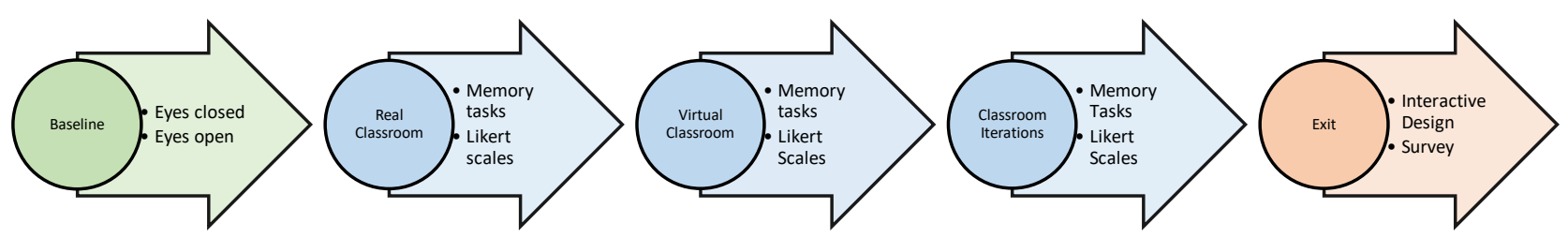

Figure 3: Experimental timeline with distinct segments of the study indicated by color. Source: authors.

angle in Grasshopper 3D and Rhinoceros. No prior knowledge of either program was expected of participants; they were shown how to rotate their view of the building and instructed to toggle sliders for each of characteristics. Participants were given a final survey at the culmination of the study.

The ten classroom designs were selected variations of significant interior features such as color, height, width, roundness and incorporation of natural elements. The first perfectly replicated the real classroom participants experienced at the beginning of the study.

All analysis of collected signals was conducted using open source EEGLAB software (Delorme and Makeig, 2004) and other MATLAB functions related to LSL. The $\mathrm{H}-\infty$ filtering program (Kilicarslan 2016) was used to initially pre-process the EEG and EOG signals and eliminate ocular artifacts. The data was subsequently preprocessed following a modified PREP pipeline (BigdelyShamlo 2015) and band-pass filtered between 0.1 and $100 \mathrm{~Hz}$ before processing according to independent component analysis and dipole fitting.

Each additional biometric signal collected was individually band-pass or high-pass filtered. From there, values such as heart rate, heart rate variability, average GSR power and average magnitude of acceleration were calculated to be compared.

\section{RESULTS}

At this stage, we have completed a pilot study with eight individual and are working on collecting the data for the main study. In this section we briefly explain out first data analysis comparing three learning environment. Our independent variables in design of this room were height of classroom, view to nature, and room texture. We analyzed the effect of these variables on learning performance of the participants during the scanning session separately. The SAM Test demonstrated that change in the design element had a significant effect on learning performance, $Z=-1.32, P<0.05$. Specifically, participants were more likely to have better learning performance if they had natural light with view to nature comparing to the room without windows $Z=-1.27, P=$ 0.021. Following the completion of EEG recording, participants were presented with all of the stimuli that they had viewed in the scanner once again, and asked to rate each stimulus on pleasantness (using a five-point scale with anchors "very unpleasant" and "very pleasant") and on learning (using a five-point scale with anchors "not learning-friendly at all" and "very learning-friendly").
Nonparametric partial correlation was computed to determine the relationship between design and the learning performance whilst controlling for pleasure. There was a positive significant partial correlation between classrooms with more simple environment comparing to the one with full texture $(p=0.037)$. However, classroom with higher ceiling did not show an impact on theta activity.
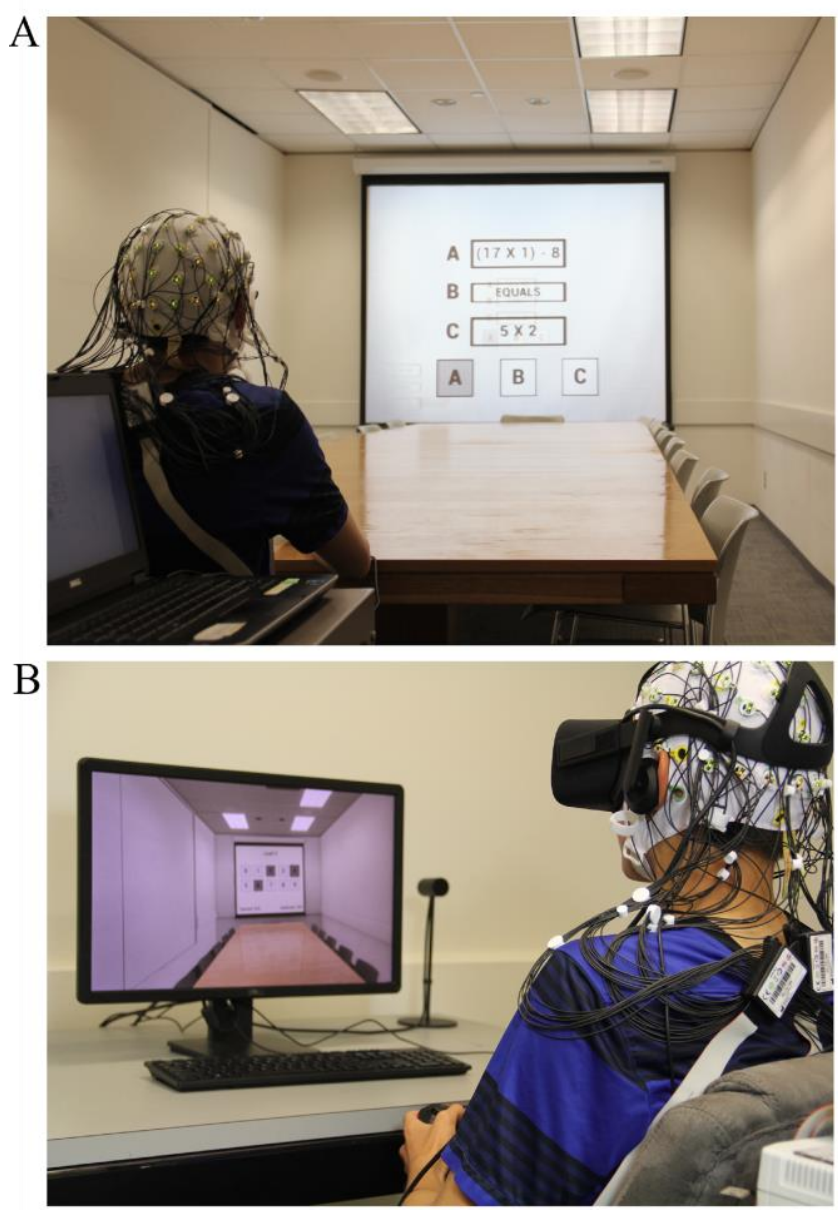

Figure 4: The research participants completed learning tasks in (a) the real classroom and (b) a virtual rendering of the classroom. Source: authors. 


\section{IMPACT ON SOCIETY AND ON THE FUTURE OF DESIGN}

This study has the potential to provide designers, educators, and psychologists with an important toolset for evaluating the relationship between architectural form and human experience. It can also provide valuable data to help neuroscientists understand cognitive reactions to spatial experience. Sociologists may be interested in using our data to evaluate relationships between demographic variables (ethnicity, nationality, socioeconomic background, etc.) and cognitive responses to architecture. Engineers may be interested in viewing our prototype as inspiration for the design of nextgeneration, context-aware, brain-body imaging (MoBI) technology.
Last but not least, the construction of a broad, multi-modal amalgamated dataset based on comparative design studies using our system could contribute significantly to the optimization of architectural design and improvements in the human quality of our built environment. The ultimate benefit to the public will be in the form of improved health, creativity, productivity, and a more satisfying architectural experience that can come from better human-centered design (Kalantari, 2017). By including demographic variables in the analysis, designers can become more aware of the effects of the built environment on specific populations, including disabled individuals, women, and other minority groups.

\section{CONCLUSION}
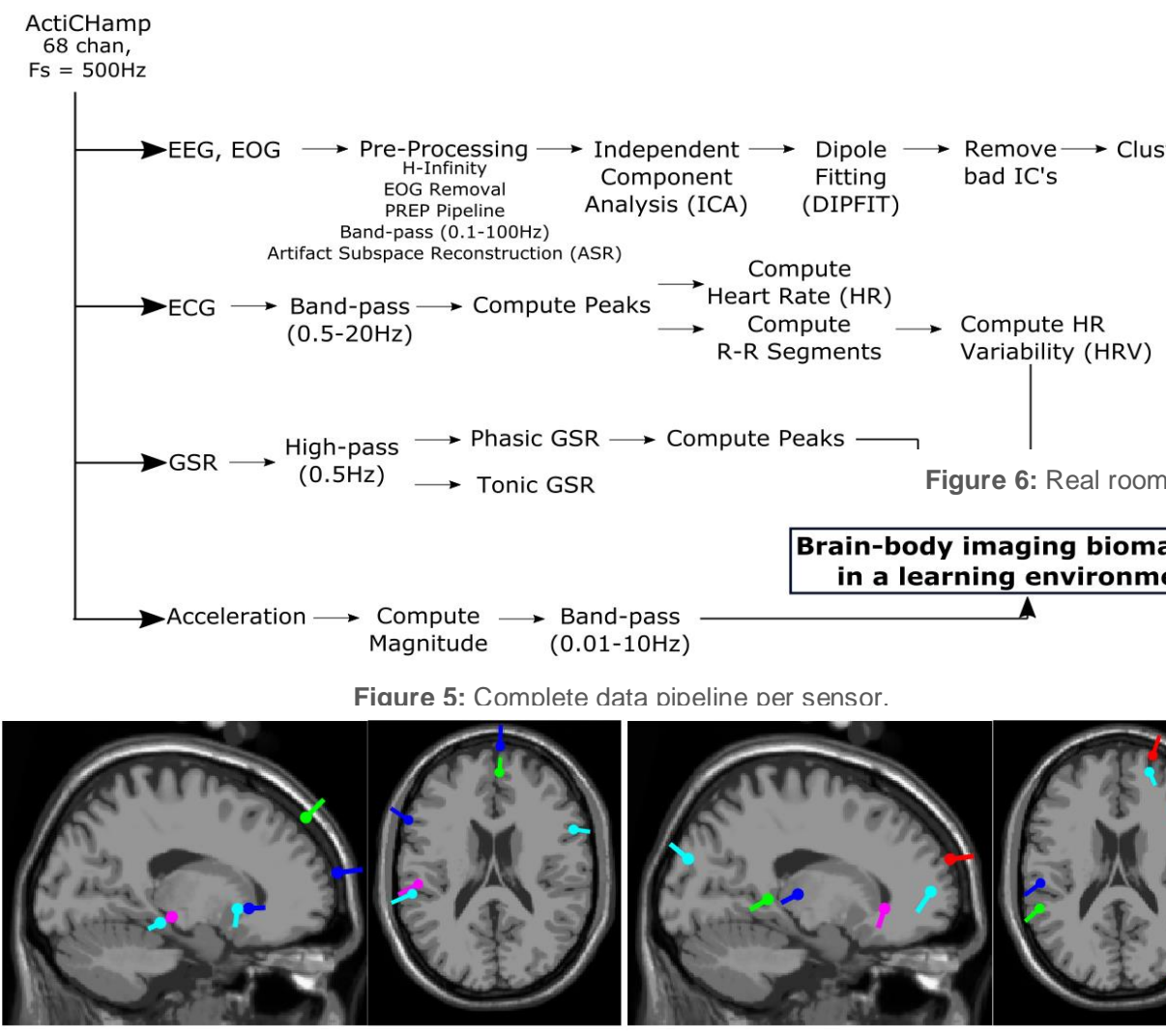

Figure 6: Real room dipoles, left, vs. virtual room dipoles,

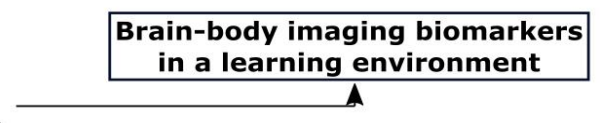

Baseline

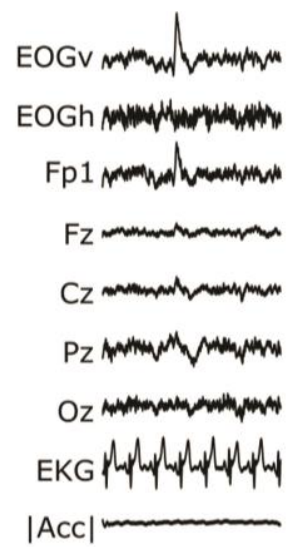

Eyes closed
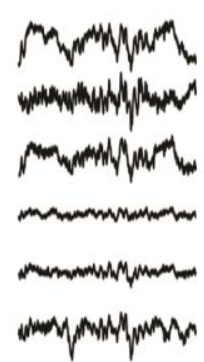

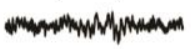

HWhythly
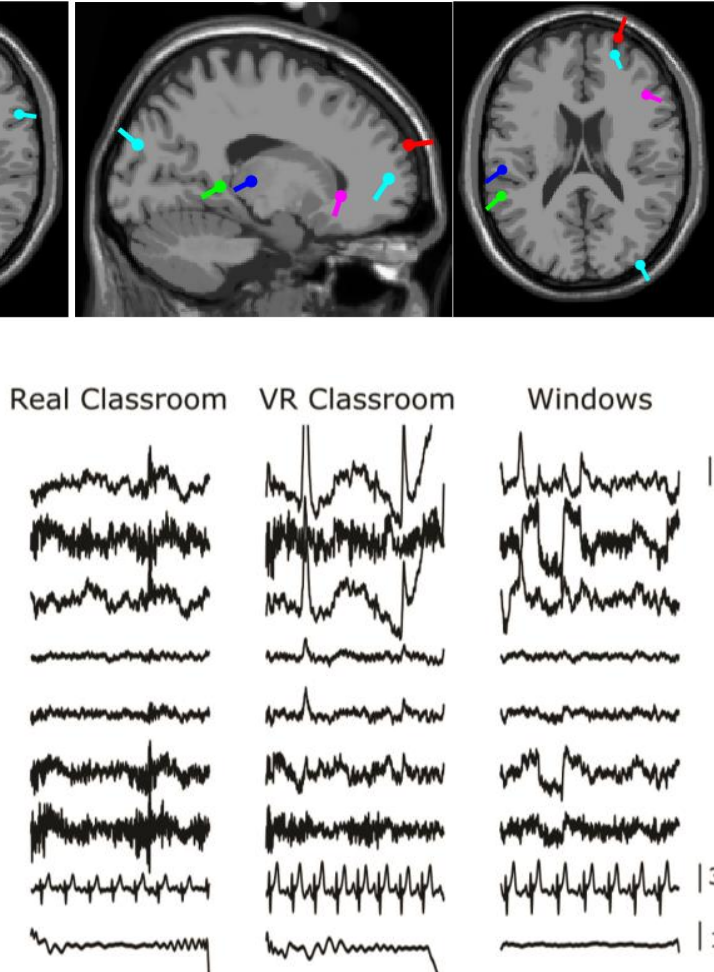

$\overline{1 \text { second }}$

Figure 7: Data obtained from the pilot study participant for five conditions (indicated in columns): baseline eyes open, baseline eyes closed, real classroom, virtual classroom, and virtual classroom with added windows. The figure rows show the initial $5 \mathrm{~s}$ of data from selected EEG, EOG, EKG and head-acceleration channels. 

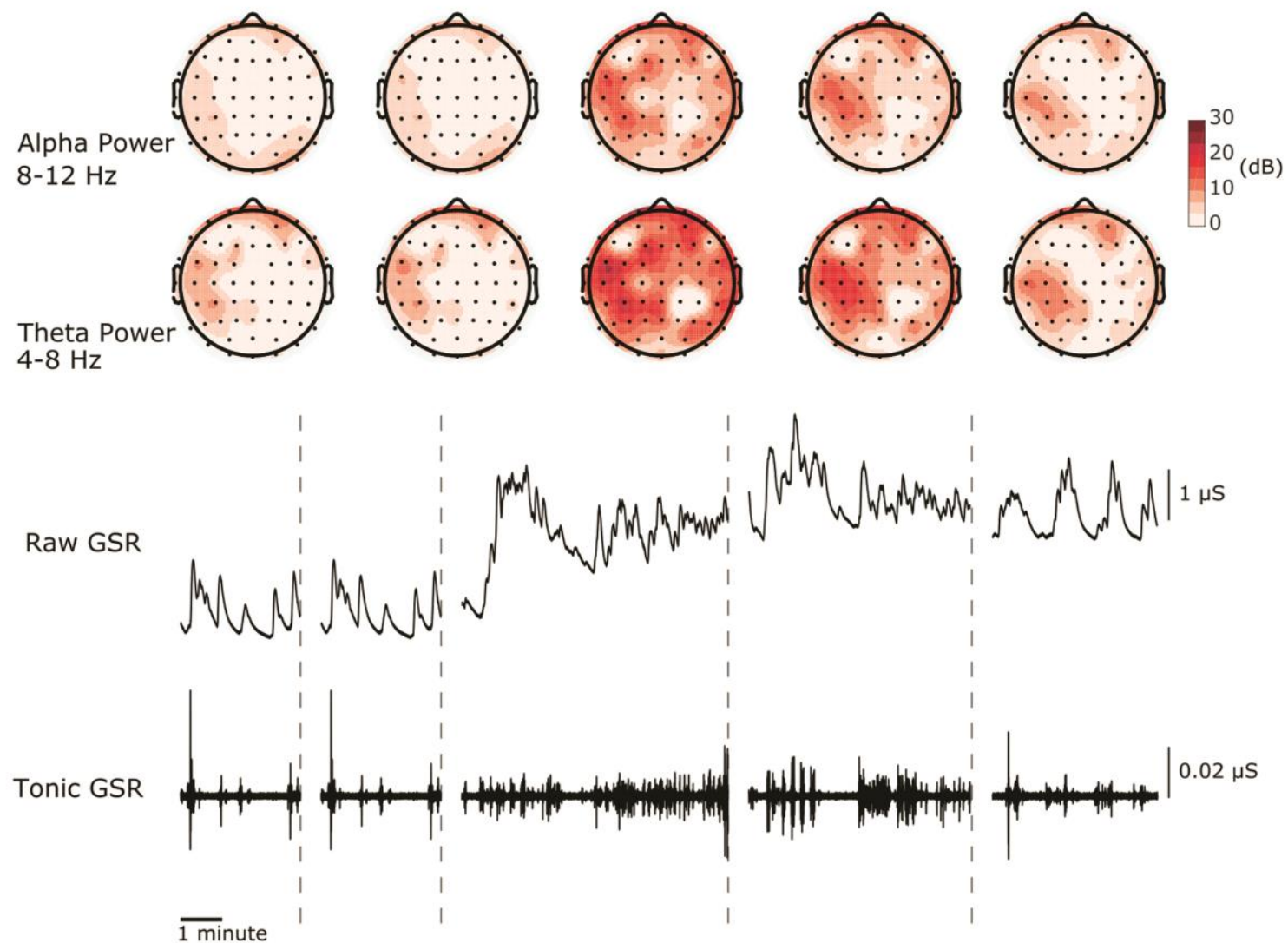

Figure 8: Data obtained from the pilot study participant for five conditions (indicated in columns): baseline eyes open, baseline eyes closed, real classroom, virtual classroom, and virtual classroom with added windows. The figure rows show (top) total al pha $(8-12 \mathrm{~Hz})$ and theta $(4-8 \mathrm{~Hz})$ power in all EEG channels, and (bottom) raw and tonic GSR (skin conductivity) signals.

A

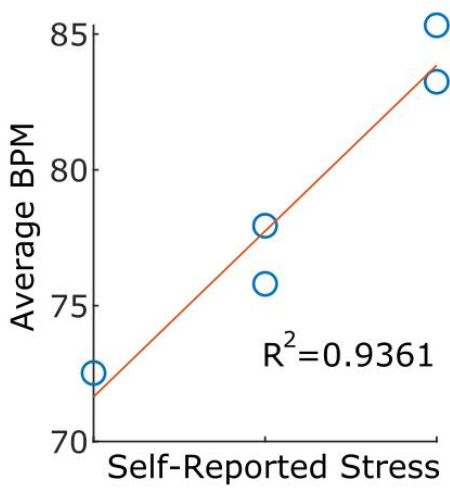

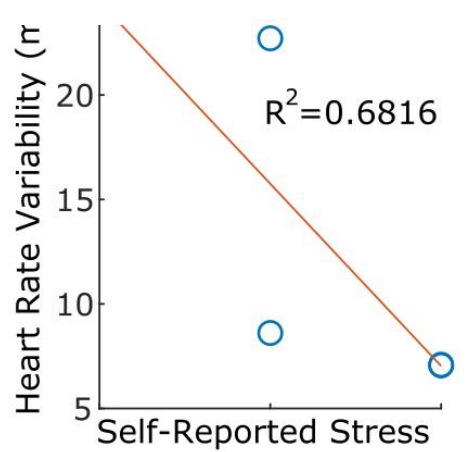

Figure 9: Correlation analysis between different non-neural biomarkers. Source: authors.

This project provides a practical toolset to test the potential human impacts of architectural design innovations. The research responds to a growing call in the field for evidence-based design and for an and for a and for an inexpensive means of evaluating the potential human effects of new designs. Our research will address this challenge by developing a prototype mobile brainbody imaging interface that can be used in conjunction with virtual immersion. This allows participants' conscious and unconscious reactions to new architectural designs to be evaluated before the buildings' physical construction.

To test the idea, we have conducted several pilot studies. In these experiments, we evaluated biometric data obtained from participants who "walked" through an architectural space in a Virtual Reality construct. We analyzed the data (which included participant demographic information), to determine if any broad and useful conclusions could be drawn about human responses to different building designs on the basis of a virtual experience. The results of the experiment indicated a significant relationship between different virtual architectural forms and measured stress levels.

Current information technology has allowed many fields to benefit from "big data" analysis in their optimization of resources. However, design fields are somewhat lacking in this area, due to the difficulty of obtaining quantitative data about human responses to design and the tremendous investment required to construct and test new architectural ideas. This study has the potential to provide designers, educators, and psychologists with an important 
toolset for evaluating the relationship between architectural form and human experience. The construction of a broad, amalgamated data-set based on these evaluations could contribute significantly to the optimization of design and the quality of our built environment. By including demographic variables in the analysis, designers can become more aware of the effects of the built environment on specific populations, including disabled individuals, women, and other minority groups.

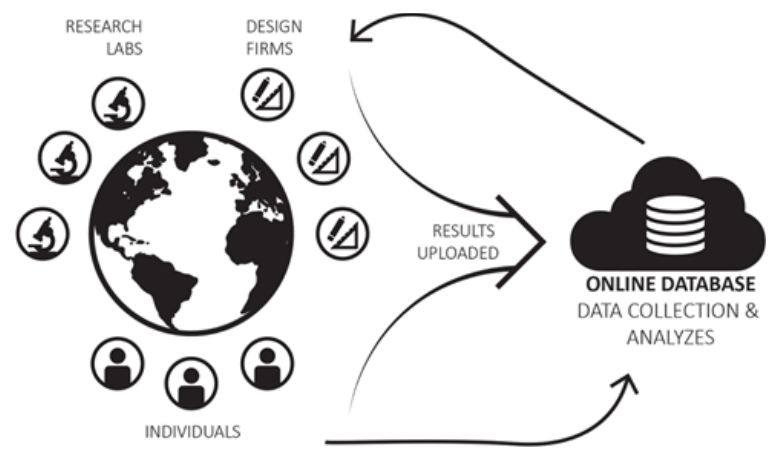

Figure 10: Broader Impact of the Research.

\section{REFERENCES}

Bigdely-Shamlo, N., Mullen, T., Kothe, C., Su, K.M. and Robbins, K.A., 2015. The PREP pipeline: standardized preprocessing for large-scale EEG analysis. Frontiers in neuroinformatics, 9, 16.

Brooks, R., Fuller, A., \& Waters, J. L. 2012. Changing spaces of education: new perspectives on the nature of learning. Routledge.

Butin, D. 2000. Multipurpose Spaces, National Clearinghouse for Educational Facilities, Washington, DC.

Chan, T. C. \& Richardson, M. D. 2005. Ins and outs of schoo facility management: More than bricks and mortar. Lanham, MD: Scarecrow Education.

Cruz-Garza, J. G., Brantley, J. A., Nakagome, S., Kontson, K., Megjhani, M., Robleto, D., \& Contreras-Vidal, J. L. 2017. Deployment of Mobile EEG Technology in an Art Museum Setting: Evaluation of Signal Quality and Usability. Frontiers in Human Neuroscience, 11, 527.

Daisey J, Angell W, Apte M. 2003. Indoor air quality, ventilation and health symptoms in schools: an analysis of existing information. Indoor Air;13:53e64.

Delorme, A., and Makeig, S. 2004. EEGLAB: an open source toolbox for analysis of single-trial EEG dynamics including independent component analysis. J. Neurosci. Methods 134, 9-21.

Fisher A, Godwin K, Seltman H. 2014, Visual environment, attention allocation, and learning in young children: when too much of a good thing may Be bad. Psychol Sci;25(7):1362e70. http://dx.doi.org/10.1177/0956797614533801.

Geller, A. L. 2003. Smart growth: a prescription for livable cities. American journal of public health, 93(9), 1410-1415.

Hansmann, R., Hug, S. M., \& Seeland, K. 2007. Restoration and stress relief through physical activities in forests and parks. Urban Forestry \& Urban Greening, 6(4), 213-225.

Healey, J. A., R. W. Picard., 2005. Detecting stress during realworld driving tasks using physiological sensors. IEEE Transactions on Intelligent Transportation Systems 6, 156166.

Jackson, R. J., \& Kochtitzky, C. 2001. Creating a healthy environment. The Impact of the Built Environment on Public Health. Washington, DC: Sprawl Watch Clearinghouse.
Kalantari, S., \& Snell, R. (2017). Post-Occupancy Evaluation of a Mental Healthcare Facility Based on Staff Perceptions of Design Innovations. HERD: Health Environments Research \& Design Journal, 10(4), 121-135.

Kaplan, S. 1995. The restorative benefits of nature: Toward an integrative framework. Journal of environmental psychology, 15(3), 169-182. Kaup, M. L., Kim, H. C., \& Dudek, M. 2013. Planning to Learn: The Role of Interior Design in Educational Settings. International Journal of Designs for Learning, 4(2).

Kilicarslan, Atilla, Robert G. Grossman, and Jose Luis ContrerasVidal. "A robust adaptive denoising framework for real-time artifact removal in scalp EEG measurements." Journal of Neural Engineering, 13:2.

Kothe, Christian. Lab Streaming Layer (LSL). Swartz Center for Computational Neuroscience 2014. Available online at: http://github.com/sccn/labstreaminglayer

Kuller R, Mikellides B, Janssens J. 2009. Color, arousal, and Performance A comparison of three experiments. Colour Res Appl; 34(2):141e52

Martin, J. L. 2010. Determining eligibility under Section 405: Fundamentals and new challenge areas. In The Council of Educators for Students with Disabilities, Inc. Retrieved from http://www.504idea.org/Council_Of_Educators/Resources_fil es/Modern\%20504\%20Eligibility.pdf

Mathews, J., Berrett, D., \& Brillman, D. 2005. Other winning equations. Newsweek, 145(20), 58-59.

McGregor, J. 2004. Spatiality and the place of the material in schools, Pedagogy, Culture and Society, Vol. 12, No. 3, pp. 347-372.

Sallis, J. F., Cervero, R. B., Ascher, W., Henderson, K. A., Kraft, M. K., \& Kerr, J. 2006. An ecological approach to creating active living communities. Annu. Rev. Public Health, 27, 297322.

Shemesh, Avishag, Ronen Talmon, Ofer Karp, Idan Amir, Moshe Bar \& Yasha Jacob Grobman 2017. Affective response to architecture - investigating human reaction to spaces with different geometry. Architectural Science Review, 60:2 (116125). DOI: $10.1080 / 00038628.2016 .1266597$

Temple, P. 2007. Learning Spaces for the 21st Century: A Review of the Literature, Centre for Higher Education Studies, Institute of Education, University of London.

Truong, K., Ma, S. 2006. A systematic review of relations between neighbourhoods and mental health, Journal of Mental Health Policy Economics, 9, 137-154.

Ulrich C. 2004. A place of their own: children and the physical environment. Hum Ecol October; 32(2):11e4.

Ulrich, R. S., Simons, R. F., Losito, B. D., Fiorito, E., Miles, M. A., \& Zelson, M. 1991. Stress recovery during exposure to natural and urban environments. Journal of environmental psychology, 11(3), 201-230.

Wargocki P, Wyon DP. 2007. The effects of moderately raised classroom temperature and classroom ventilation rate on the performance of schoolwork by children (1257-RP). HVAC\&R Res; 13(2):193e220.

Wheaton, B., Nisenbaum, R., Glazier, R. H., Dunn, J. R., \& Chambers, C. 2015. The neighbourhood effects on health and well-being (NEHW) study. Health \& place, 31, 65-74.

Woolner, P., Hall, E., Higgins, S., McCaughey, C., \& Wall, K. 2007. A sound foundation? What we know about the impact of environments on learning and the implications for Building Schools for the Future. Oxford Review of Education, 33(1), 47-70.

Zeisel J, Silverstein NM, Hyde J, Levkoff S, Lawton MP, Holmes W. 2003. Environmental correlates to behavioral health outcomes in Alzheimer's special care units. Gerontologist; 43(5):697e711 